\title{
FISSION PROPERTIES OF NEUTRON RICH URANIUM ISOTOPES
}

\author{
Samuel A. Giuliani ${ }^{* \dagger}$ \\ Institut für Kernphysik (Theoriezentrum), Technische Universität Darmstadt, \\ Schlossgartenstraße 2, 64289 Darmstadt, Germany. \\ E-mail: giuliani@theorie.ikp.physik.tu-darmstadt.de
}

\section{Gabriel Martínez-Pinedo}

Institut für Kernphysik (Theoriezentrum), Technische Universität Darmstadt,

Schlossgartenstraße 2, 64289 Darmstadt, Germany.

E-mail: martinez@theorie.ikp.physik.tu-darmstadt.de

\section{Luis Miguel Robledo}

Departamento de Física Teórica, Universidad Autónoma de Madrid, E-28049 Madrid, Spain.

E-mail: luis.robledo@uam.es

\begin{abstract}
Fission properties of 330 heavy and superheavy nuclei are computed using the Barcelona-CataniaParis-Madrid energy-density functional. Potential energy surfaces as well as collective inertias relevant to the fission process are obtained within a mean-field approach. Spontaneous fission half-lives are computed using the semiclassical Wentzel-Kramers-Brillouin formalism. As tracks of possible magic numbers in the superheavy region, combinations of neutron and proton number leading to an enhanced stability against the spontaneous fission process are discussed. For Uranium isotopes, the agreement with other theoretical models is also studied.
\end{abstract}

XIII Nuclei in the Cosmos

7-11 July, 2014

Debrecen, Hungary

* Speaker.

$\dagger$ A footnote may follow. 


\section{Introduction}

Fission is a crucial phenomenon to understand r-process nucleosynthesis [1-3]. Metal-poor star observations suggest a very robust r-process abundance pattern for elements heavier than $Z \sim$ 50 and the most likely reason to achieve such a robust pattern is fission cycling. The evolution of the nucleus from its ground state to the scission point is driven by a delicate balance between nuclear force binding nucleons together and Coulomb repulsion. But despite of its discovery more than 70 years ago, the fission process is still far from being well understood.

In the last decade a great number of studies were devoted to the description of the fission process within the Density Functional Theory (DFT). This mean-field approach based in the HartreeFock-Bogoliubov (HFB) theory requires for a phenomenological effective interaction fitted to reproduce nuclear matter properties and/or equations of state. The large variety of papers studying fission properties of Skyrme [4-6], Gogny [7-9], and relativistic mean-field based [10-12] interactions or pure energy density functionals [13] denotes the important effort made towards a better description of the fission process within the Energy Density Functional (EDF) theory.

The Barcelona-Catania-Paris-Madrid (BCPM) [13] is a newly proposed EDF inspired by the Kohn-Sham theory. Its free parameters were adjusted in order to reproduce the binding energy of the 518 even-even nuclei of the Audi and Wapstra 2003 mass table evaluation. In a recent paper [14] the fission properties of the BCPM EDF were compared with available experimental data. After those encouraging results, we present here an extended calculations of the spontaneous fission lifetimes, fission barrier heights and 2-neutron separation energies for 330 even-even heavy and superheavy nuclei. The general trends of this quantities are discussed in order to identify regions of stability against the spontaneous fission process.

\section{Methodology}

The BCPM interaction is a so called "pure" energy-density functional which bulk part is given by a polynomial fit to realistic equations of state in both neutron and symmetric nuclear matter. These polynomial fits are then translated into finite nuclei using the Local Density Approximation. In addition, finite size effects are taken into account by introducing a phenomenological finiterange interaction. The open shell-nuclei pairing effects are described using a zero-range density dependent interaction, fitted to reproduce the nuclear matter gaps obtained with the Gogny force [15]. The remaining contributions are the Coulomb interaction and a zero-range spin-orbit term.

Within the nuclear EDF, the fission process is described following the approach of the HartreeFock-Bogoliubov (HFB) theory with constraining operators. Spontaneous fission lifetimes $t_{\mathrm{Sf}}$ (in seconds) are computed using the semiclassical approach of the WKB formula:

$$
t_{\text {sf }}=2.86 \times 10^{-21}(1+\exp (2 S))
$$

where $S$ is the action integral computed along the fission path $s$ :

$$
S=\int_{a}^{b} d s \sqrt{2 B(s)\left[V(s)-\left(E_{0}+E_{G S}\right)\right]} .
$$

The fission path $s$ is determined using the traditional static prescription where the potential energy is minimized in each configuration of the multidimensional space. The parameters entering 
in the equation (2.2) are the HFB mean-field energy $V$ including the rotational correction $\varepsilon_{\text {rot }}$, the ground state energy of the nucleus $E_{G S}$ and the zero-point energy correction $E_{0}=1.0 \mathrm{MeV}$ (coming from quantal fluctuations along the collective degrees of freedom). Finally, the inertias $B(s)$ associated to the collective motion are computed in terms of the relevant coordinates $q$ along the multidimensional fission path $s$ :

$$
B(s)=\sum_{i j} B_{i j} \frac{d q_{i}}{d s} \frac{d q_{j}}{d s} .
$$

The collective inertias $B_{i j}$ are computed using the Gaussian Overlap Approximation (GOA) to the Generator Coordinate Method (GCM). Since the contributions of the octupole and hexadecapole deformations to the expression (2.3) are small, we mainly use as a constraining field the quadrupole moment operator. Reflection symmetry is allowed to break at any stage of the calculations in order to permit asymmetric fission and octupole deformation of the nucleus. Finally, in order to reduce the computational cost axial symmetry is preserved. The HFB equations are solved using a gradient-like algorithm, which allows an easy generalization to an arbitrary number of constraining operators. The computations are carried out using a modification of the HFBaxial computer code [16].

In recent papers [17-19] the sensitivity of the fission properties to the parameters uncertainties entering in the formula (2.2) was studied. We are aware of the large variability that characterizes the computation of the fission properties, specially regarding the strong dependence of the spontaneous fission lifetimes with the pairing strength. However, the uncertainties are related in most of the cases with the absolute value of the fission observables rather than their systematic behavior. BCPM proved its capability to give a good description of the fission properties in terms of general trends of fission barriers and spontaneous fission lifetimes. For this reason we used fixed values of pairing strengths and zero-point energy correction, as well as only the GCM method in the computation of the collective masses.

\section{Results}

With the aim of studying the impact of spontaneous fission (SF) on the superheavy nuclear landscape, the fission properties of 330 even-even nuclei with $92 \leq Z \leq 120$ and $160 \leq N \leq 202$ were computed using the BCPM EDF. In Fig.1a spontaneous fission lifetimes $t_{\mathrm{Sf}}$ are depicted in the $(N, Z)$ plane. The theoretical values of $t_{\mathrm{Sf}}$ show three different regions with larger stability against the SF process. The first one is located around the predicted magic number $N=184$ with very long-lived actinides isotopes $(Z=92-96)$. The second region is placed around the neutrondeficient isotopes of elements with $102 \leq Z \leq 110$. Finally, a slight increase of the $t_{\mathrm{sf}}$ is noticed in the very heavy region $Z=118,120$ around the neutron number $N=178$. These three regions are a predicted occurrence of magic shell closures [20] leading to more stable nuclei. However, it seems that in our calculations there is no mark of the hypothetical doubly magic number ${ }^{298} 114$, which at the beginning of the sixties was predicted to be the center of a possible island of stability [21]. From the results of the $t_{\mathrm{sf}}$ it is possible to infer that the magicity of a particular neutron(proton) number depends on the proton(neutron) number present in the nucleus.

The general trend of $t_{\mathrm{sf}}$ in Fig.1a can be partially explained by the magnitude of the fission barrier. In Fig.1b the highest fission barrier height of each nuclei are plotted in the $(N, Z)$ plane. 


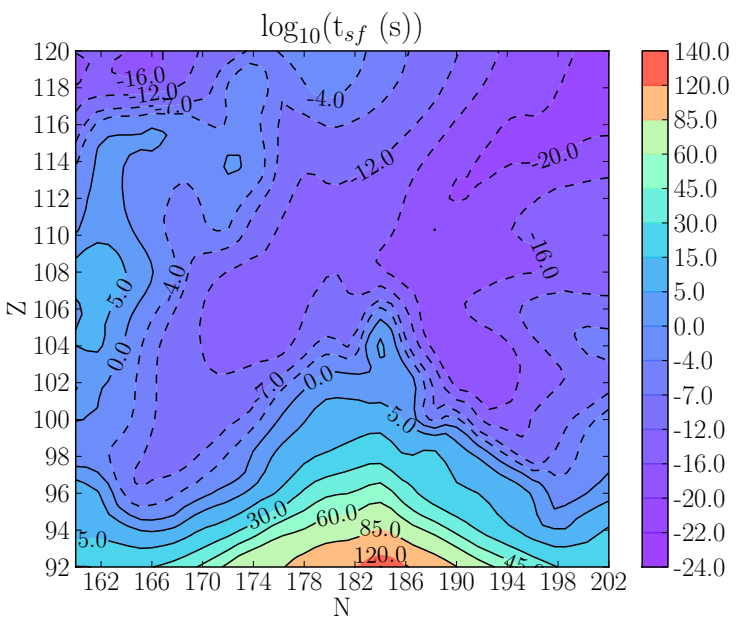

(a) $\log _{10}\left(t_{\mathrm{sf}}[s]\right)$

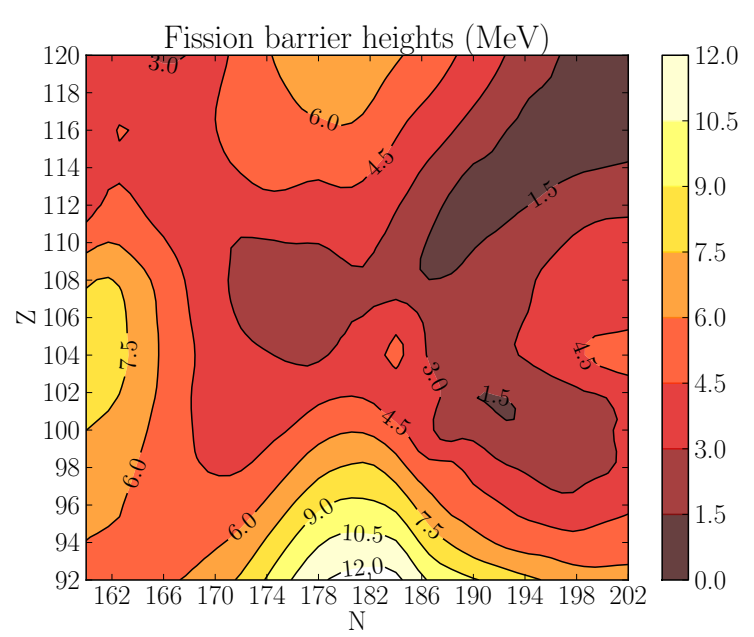

(b) Fission barrier heights $[\mathrm{MeV}]$

Figure 1: Contour plot in the $(N, Z)$ plane of (a) the spontaneous fission lifetimes and (b) the maximum fission barrier heights predicted by BCPM. Dashed lines mean a negative value of $t_{\mathrm{sf}}$.

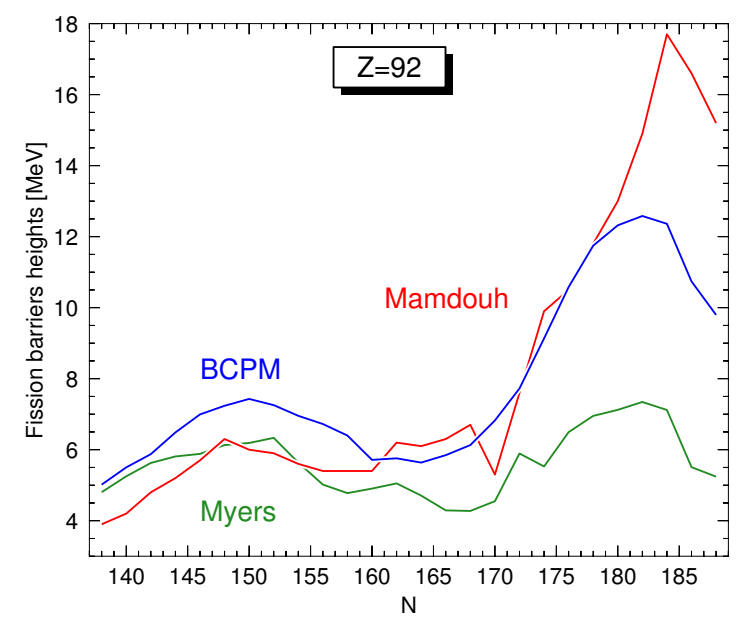

(a) Fission barrier heights in $\mathrm{MeV}$ predicted by the BCPM (blue line), the ETFSI [22] (red line) and the TF [21] (green line) models.

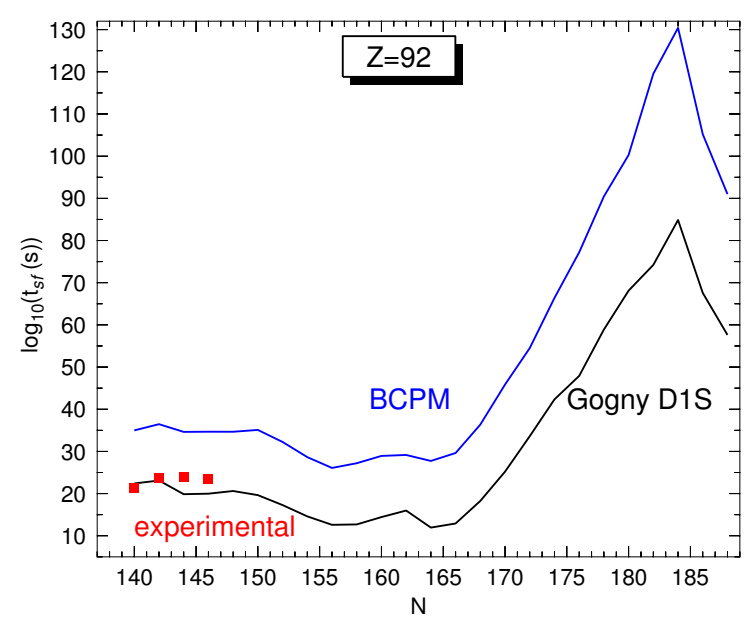

(b) Logarithm of the spontaneous fission lifetimes predicted by BCPM (blue line) and the Gogny D1S interaction [9] (black line). Experimental values (red squares) are taken from [24].

Figure 2: Fission properties of even-even Uranium isotopes.

In the three regions mentioned above the fission barrier heights are clearly higher compared to the rest of the nuclei. The increase of the barrier heights can be a signal of larger stability against the spontaneous fission process, even though other components of the potential energy surface can also affect the value of $t_{\mathrm{sf}}$ (e.g. the number of fission barriers or the stretching of the potential energy tail). In Fig. 2 the BCPM fission properties are compared with other theoretical predictions along the Uranium isotopic chain. In Fig.2a, fission barrier heights taken from the BCPM, the Extended Thomas Fermi with Strutinsky Integral (ETFSI, [22]) and the Thomas-Fermi (TF, [23]) model 


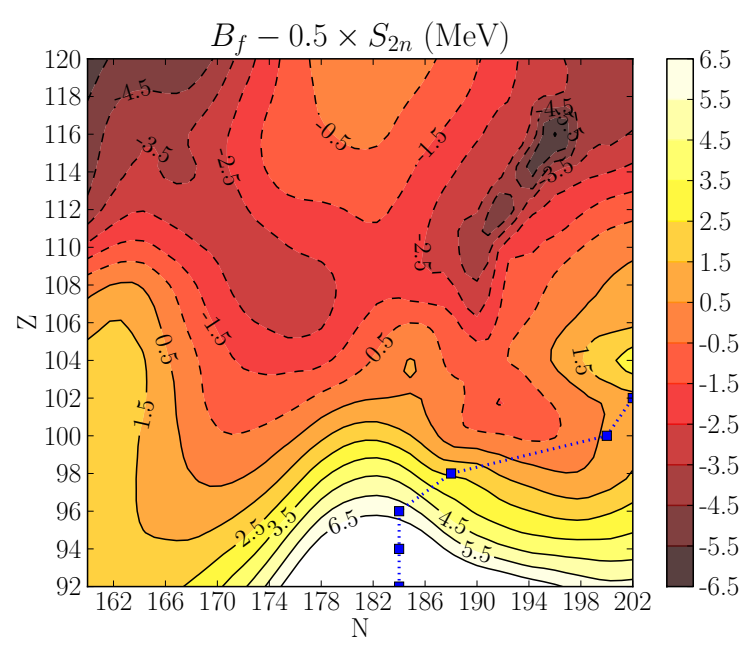

Figure 3: Contour plot of the quantity $R_{B S} \equiv B_{f}-0.5 \times S_{2 n}$ in $\mathrm{MeV}$ computed with the BCPM interaction, where dashed lines mean a negative value of $R_{B S}$. Blue squares represent the heaviest isotope for each nuclei with $Z \leq 102$ and $S_{2 n} \geq 4.0 \mathrm{MeV}$.

are depicted as a function of the neutron number $N$. For lightest isotopes, both TF and ETFSI values agree quite well while BCPM predicts slightly larger barriers. For the heaviest isotopes the three models differs quite strongly, with BCPM predicting an intermediate value of the barrier heights. In Fig.2b the $t_{\mathrm{sf}}$ values of BCPM and the Gogny D1S parametrization [9] are depicted as a function of the neutron number $N$. The general trend of $t_{\mathrm{sf}}$ with $N$ of both models is similar all along the Uranium isotopic chain. The D1S parametrization predicts lower values of $t_{\mathrm{sf}}$ than BCPM, and its results are in better agreement with the experimental data from [24]. This result was expected since the D1S parametrization uses fission data in its fitting protocol. However, the fact that BCPM reproduces the general behavior of the D1S interaction gives a great confidence regarding its predicting power.

One quantity of particular interest for studying the neutron-induced fission cross sections is the difference between the fission barrier height $B_{f}$ and the neutron separation energy $S_{n}$ [25]. This quantity determines where the production of superheavy elements during the r-process can be inhibited by the neutron-induced fission process $\left(B_{f}-S_{n} \leq 0 \mathrm{MeV}\right)$. As a qualitative analysis of this quantity, in Fig.3 the $R_{B S} \equiv B_{f}-0.5 \times S_{2 n}$ value is depicted in the $(N, Z)$ plane. For rough estimations it is usually considered $R_{B S} \leq 2 \mathrm{MeV}$ as the limit in the competition between neutron capture and neutron-induced fission. This means that nuclei with a lower value of $R_{B S}$ would fission immediately after capturing a neutron. As a consequence of this estimation, we can conclude that after the magic shell $N=184$ the production of heavier nuclei is inhibited by the neutron-induced fission. This results agree with the predictions made in Ref. [2] using a combination of the TF barriers and FRDM masses.

\section{Conclusions}

The fission properties of 330 even-even nuclei were computed using the BCPM energy density functional. Even though the results do not show a clear track of magic neutron or proton number all 
along the superheavy nuclear landscape, spontaneous fission lifetimes and fission barriers heights indicate three different regions of enhanced stability against the spontaneous fission process. All these regions were predicted to be a possible combination of neutron and proton number leading to magic shell closures. For actinides nuclei close to the neutron drip-line, a general enhancement of the stability against the SF is also predicted.

Studying the difference between fission barriers heights and 2-neutron separation energy we found that the only region where the r-process can proceed towards the formation of superheavy elements is located around $92 \leq Z \leq 102$ and $170 \leq N \leq 202$. In the rest of the chart the nuclei are expected to fission immediately after capturing a neutron.

Fission yields computations are required for a complete nucleosynthesis modeling, as well as calculations of the fission properties of nuclei with an odd number of protons and/or neutrons. This work is already in progress.

\section{Acknowledgments}

We are thankful to R. Rodríguez-Guzmán for providing the Gogny results shown in Fig.2b. The work of SAG and GMP was supported by the Helmholtz Association through the Nuclear Astrophysics Virtual Institute (VH-VI-417) and the BMBF-Verbundforschungsprojekt number 06DA7047I. The work of LMR was supported by the Spanish MICINN Grants No. FPA2012-4694 and No. FIS2012-34479 and by the Consolider-Ingenio 2010 MULTIDARK CSD2009-00064.

\section{References}

[1] G. Martínez-Pinedo, D. Mocelj, N. T. Zinner, A. Kelić, K. Langanke, I. V. Panov, B. Pfeiffer, T. Rauscher, K. H. Schmidt and F.-K. Thielemann, The role of fission in the r-process, Prog. Part. Nucl. Phys. 59, 199 (2007).

[2] I. Petermann, K. Langanke, G. Martínez-Pinedo, I. V. Panov, P.-G. Reinhard and F.-K. Thielemann, Have superheavy elements been produced in nature? Eur. Phys. J. A48, 122 (2012).

[3] I. V. Panov, I. Y. Korneev, G. Martínez-Pinedo, and F.-K. Thielemann, Influence of spontaneous fission rates on the yields of superheavy elements in the r-process Astron. Lett. 39, 150 (2013).

[4] N. Nikolov, N. Schunck, W. Nazarewicz, M. Bender and J. Pei, Surface symmetry energy of nuclear energy density functionals, Phys. Rev. C83 034305 (2011).

[5] J. Erler, K. Langanke, H.P. Loens, G. Martínez-Pinedo, and P.-G. Reinhard, Fission properties for r-process nuclei, Phys. Rev. $\mathbf{C 8 5} 025802$ (2012).

[6] J. McDonnell, N. Schunck and W. Nazarewicz, Fission Properties of Neutron-Rich Nuclei, World Scientific, Singapore, p. 597 (2013).

[7] J.F. Berger, M. Girod and D. Gogny. Microscopic analysis of collective dynamics in low energy fission Nucl. Phys. A428, 23c (1984).

[8] J.L. Egido and L.M. Robledo, Fission Barriers at High Angular Momentum and the Ground State Rotational Band of the Nucleus ${ }^{254}$ No Phys. Rev. Lett. 85, 1198 (2000).

[9] R. Rodríguez-Guzmán and L.M. Robledo Microscopic description of fission in uranium isotopes with the Gogny energy density functional Phys. Rev. C89, 054310 (2014). 
[10] H. Abusara, A.V. Afanasjev and P. Ring, Phys. Rev. C82, 044303 (2010).

[11] B.N. Lu, E.G. Zhao and S.G. Zhou, Phys. Rev. C85, 011301 (2012).

[12] A.V. Afanasjev and O. Abdurazakov, Pairing and rotational properties of actinides and superheavy nuclei in covariant density functional theory Phys. Rev. C88, 014320 (2013).

[13] M. Baldo, L.M. Robledo, P. Schuck and X. Viñas, Physical Review C87, 064305 (2013).

[14] S. A. Giuliani and L. M. Robledo, Fission properties of the Barcelona-Catania-Paris-Madrid energy density functional Phys. Rev. C88, 054325 (2013).

[15] E. Garrido, P. Sarriguren, E. Moya de Guerra, and P. Schuck, Effective density-dependent pairing forces in the $T=1$ and $T=0$ channels, Phys. Rev. C60, 064312 (1999).

[16] L.M. Robledo, HFBaxial computer code (2002).

[17] J. Sadhukhan, K. Mazurek, A. Baran, J. Dobaczewski, W. Nazarewicz and J. Sheikh, Spontaneous fission lifetimes from the minimization of self-consistent collective action Phys. Rev. C88, 064314 (2013).

[18] R. Rodríguez-Guzmán and L. M. Robledo, Microscopic description of fission in neutron-rich plutonium isotopes with the Gogny-D1M energy density functional Eur. Phys. J. A50, 142 (2014).

[19] S. A. Giuliani, L. M. Robledo and R. Rodriguez-Guzman, Dynamic versus static fission paths with realistic interactions, arXiv:1408.6940 [nucl-th].

[20] T. Sil, S. Patra, B. Sharma, M. Centelles and X. Viñas, Superheavy nuclei in a relativistic effective Lagrangian model Phys. Rev. C69, 044315 (2004).

[21] W. D. Myers and W. J. Swiatecki, Nuclear masses and deformations, Nucl. Phys. A81, 1 (1966).

[22] A. Mamdouh, J. M. Pearson, M. Rayet, and F. Tondeur, Fission barriers of neutron-rich and superheavy nuclei calculated with the ETFSI method, Nucl. Phys. A679, 337 (2001).

[23] W. D. Myers and W. J. Swiatecki, Nuclear properties according to the Thomas-Fermi model, Nucl. Phys. A601, 141 (1996).

[24] K.-H. Schmidt, S. Steinhäuser, C. Böckstiegel, A. Grewe, A. Heinz, A. R. Junghans, J. Benlliure, H.-G. Clerc, M. de Jong, J. Müller, M. Pfützner and B. Voss, Relativistic radioactive beams: A new access to nuclear-fission studies Nucl. Phys. A665, 221 (2000).

[25] I. V Panov, I. Y. Korneev, T. Rauscher, G. Martínez-Pinedo, A. Kelić-Heil, N. T. Zinner and F.-K. Thielemann, Neutron-induced astrophysical reaction rates for translead nuclei, Astron. Astrophys. 513, A61 (2010). 